\title{
Parameter Estimation for Towed Cable Systems Using Moving Horizon Estimation
}

Liang Sun, Jeremy Castagno, John D. Hedengren, and Randal W. Beard

L. Sun is with Academy Center for UAS Research, US Air Force Academy, CO, 80840, USA. Email: solbuaa@gmail.com.

J. Castagno and J. D. Hedengren are with the Department of Chemical Engineering, Brigham Young University, Provo, UT 84602, USA.

R. W. Beard is with the Department of Electrical and Computer Engineering, Brigham Young University, Provo, UT 84602, USA. 


\title{
Parameter Estimation for Towed Cable Systems Using Moving Horizon Estimation
}

\begin{abstract}
This paper presents a strategy for optimal estimation of parameters for towed cable systems using moving horizon estimation (MHE). The main contributions of the work include a novel formulation of MHE using a dead-band that explicitly rejects measurement noise, real-time implementation results, and the investigation of time varying stochastic disturbances as well as unknown yet constant disturbances. Further analysis is conducted on the observability and sensitivity of key parameters to determine which parameters can be estimated by the proposed approach using realtime streaming data from experiments. In addition to the real-time results, an offline multi-objective optimization is conducted to reveal the tradeoff between the computational burden and estimation variation. A Pareto frontier is used to demonstrate a series of optimal experimental configurations obtained by adjusting the model complexity (number of cable links and horizon steps). Finally, a multivariate parameter estimation is performed to explore the applicability of the proposed approach in simultaneously estimating multiple parameters.
\end{abstract}

\section{Index Terms}

Unmanned Aerial Vehicle (UAV), Parameter estimation, towed cable system, moving horizon estimation, aerial recovery, multi-objective optimization.

\section{INTRODUCTION}

The ability of a cable to transmit forces and electrical signals over great distances allows users to extend their influence to the remote region inaccessible to humans. In the past decades, towed cable systems have been studied in various applications. Such applications include payload delivery and pickup [1-3], kites [4], aerostats [5], towing bodies [6, 7], terrain following [8] and aerial refueling systems [9]. A typical towed cable system, as depicted in Figure 1, includes three components: a towing vehicle, a cable (string or tether) and a towed body (drogue). Depending on the working environment, the towing vehicle is typically an aircraft or a vessel, and the cable is typically a long, thin, and relatively light-weight material connecting the towing vehicle and the towed body, which is a device with large drag relative to the cable and a small dimension and weight relative to the towing vehicle.

Previous studies of towed cable systems can be classified into topics like stability and equilibrium analysis [1, 5 , 10-12], mathematical modeling [5, 11, 13], model validation [14-16], and control strategy development [2, 7, 8, 17]. The work related to the aerial recovery project in which a towed cable system was used to retrieve Miniature Air Vehicles (MAVs) can be found in [16, 18-23]. The basic concept of the aerial recovery mission is described in Figure 1, where the towing vehicle (mothership) is commanded to follow a circular path that results in an orbit of the towed body (drogue) with smaller radius and lower speed relative to the mothership. The MAV is regulated to follow the drogue orbit and to approach the drogue with a relatively low airspeed. After the MAV is captured by the drogue, the mothership retrieves the towed cable and drogue/MAV to complete the aerial recovery. Previous 


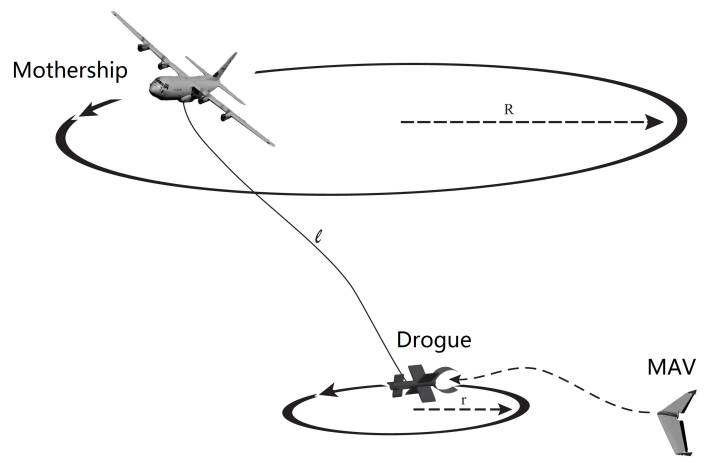

Fig. 1. The baseline concept of aerial recovery using a towed cable system.

studies for the aerial recovery system include mathematical modeling approaches using discrete models [18, 21], motion planning and control strategies [19, 20, 22, 23], and the experimental validation of both the mathematical model [16] and control strategies [22, 23] using flight tests.

In our previous work, the sensitivity of several parameters like the applied cable length, the aerodynamic drag coefficient of the drogue, and the drogue mass were studied [16]. In simulation after the flight test, the towing vehicle was forced to follow the actual mothership trajectory of the flight test. Several key parameters such as the number of cable links used in the model and the aerodynamic drag and lift coefficients of the towed body were manually tuned to match the simulation to the flight test results. Tuning parameters in this complex model is computationally demanding and requires specialized techniques to fully use the dynamic data. In the literature, the parameters used to simulate towed cable systems are typically determined by trial and error to find an acceptable match to experimental data [14-16, 24, 25]. Research focused on optimal parameter estimation for towed cable systems cannot be found, particularly for real-time applications. This paper aims to fill this gap.

The central problem in modeling towed cable systems is how the cable is treated. Methods of cable modeling can be classified into two categories: continuous methods [1, 10, 11, 25, 26] and discrete methods [12, 14, 18, 27-29]. The choice between the two categories depends on different aspects including solution accuracy, computation time, method of formulation, and personal preference [15]. This paper uses the finite element approach to represent the dynamics of the cable, which is considered as a series of $N<\infty$ elastic straight-line segments with lumped masses at the joints. Moreover, the applied forces exerted on the cable and drogue are also calculated to estimate system dynamics.

In the literature, Lagrange's method [4, 30], Kane's equations [15, 31], Gauss's Principle [18, 21], and Newton's second law $[19,20]$ have been applied to establish the equations of motion for towed cable systems. Lagrange's and Kane's approaches require explicit description of internal and external forces and do not express the system dynamics in a concise way for a large number of cable links. Methods based on Gauss's Principle are suitable for inelastic cables, which may be inappropriate for the cable (e.g. a fishing line) used in the current experiment. Newton's second law, as a fundamental tool to formulate equations of motion for dynamical systems, is applied in 
this paper to derive equations of motion for the towed cable system with a flexible and elastic cable.

Given a mathematical model and associated experimental data, an approach is needed to align the two by adjusting certain parameters in the mathematical model. In this paper, moving horizon estimation (MHE) is used to obtain optimal estimates for parameters of interest. MHE uses a receding window of prior measurements to adjust parameters and model predictions over a past time horizon. In addition, MHE allows nonlinear models and variable constraints and differs from the Kalman filter (linear models, unconstrained), the Extended Kalman filter (nonlinear models, unconstrained), the Unscented Kalman filter (nonlinear models, particle based method), or other types of estimation methods. Moreover, MHE outperforms the Extended Kalman Filter (EKF) when there are active constraints [32]. Recent advances in algorithms and computational hardware also improve the application of MHE to large-scale industrial systems [33]. Thus, MHE gives a more accurate description of the current state of the process and unmeasured disturbances [34]. An unbiased estimate (offset free) is guaranteed by adding as many input or output disturbance variables as the number of measurements [35-37].

Although MHE allows the use of nonlinear models, some simplifications such as linearized models allow faster convergence, especially where cycle time is a concern for real-time estimation of parameters [38]. MHE has been applied to improve the position and wind disturbance estimation for real-time flight navigation [39]. Simulation studies have shown the application of other nonlinear estimation methods such as particle filtering [40, 41], UKF [42] and EKF [43], but only in simulation. The development in this paper allows MHE to be communicated to flight control systems that are on-board a UAV. There is persistent communication from the UAV to a ground station where MHE is performed and the subsequent control algorithm can transmit new directives to the UAV. This ground based computing allows for more sophisticated algorithms that utilize significant computational resources for real-time flight control but would be a limitation if UAV communication is unavailable. The real-time results in this study required $0.1 \mathrm{~s}$ of clock time for each cycle of the controller. However, the cycle time was set to $1 \mathrm{~s}$ to allow for data communication requirements to the ground station and update rates on the mothership and drogue GPS devices. Embedding the algorithms in an on-board computing platform would eliminate the communication overhead but require heavier computing infrastructure on the aircraft. The optimization of this trade-off has not been adequately addressed and will be the subject of future studies.

The complex nonlinear dynamics and physical constraints of towed cable systems make it impractical to use traditional methods such as the Kalman Filter. By virtue of efficient numerical solvers, MHE is able to estimate the relevant parameters in real-time, despite using an iterative computational approach. In the literature, work has not been found that uses rigorous, nonlinear models to update a physics-based description of coordinated aircraft in real-time. This paper presents several innovations such as parameter estimates of towed cable systems given realtime measurements. These measurements are reconciled to the system constraints and nonlinear models by adjusting certain parameters. The objective of this paper is to perform real-time parameter estimation, while limited flight trials only provide results for the estimation of the applied cable length. However, the feasibility of applying the proposed approach in real-time applications is well tested. To further investigate the effectiveness of the proposed approach, subsequent comprehensive off-line estimation is conducted in simulation and the results are presented in 
this paper.

For most estimation methods, optimal estimates are obtained by using objective functions based on squared-error or $\ell_{2}$-norm formulations (e.g. Kalman filtering). To improve the accuracy and the computational efficiency of the estimation algorithm, this paper uses nonlinear dynamic equations of the system and a novel form of the objective function to formulate the MHE problem. This form takes advantage of a dead-band around current measurements in order to reject measurement noises with an $\ell_{1}$-norm function for model values that are outside of the dead-band. The additional linear set of error models can be solved by efficient numerical strategies. Continuously differentiable equations of motion are also compatible with many Nonlinear Programming (NLP) solvers.

This work is a counterpart to another paper [22] that describes an optimal trajectory generation strategy for towed cable systems, where a nonlinear model predictive control (MPC) technique is used, based on updated parameters, to obtain optimal trajectories for the towing vehicle to place the towed body onto a desired orbit in the presence of operational constraints and constant disturbances such as cross-wind. This paper details a real-time method of obtaining parameter estimates that are fed into the model for the subsequent trajectory generation using MPC.

The remainder of this paper is structured as follows. In Section II, a mathematical model for a cable-drogue system is established using Newton's second law. Section III introduces the formulation of MHE used to achieve optimal solutions for specified parameters of the system. Section IV shows the hardware platform of the flight test and real-time estimation results. Section V presents single-parameter and multivariate estimation results in off-line simulations for key parameters like the aerodynamic drag coefficient of the drogue, the Young's modulus, and the unmeasured wind component in the vertical direction.

\section{MathematicAl MODEL}

The cable used in experiments is a Nylon fishing line, which stretches and contracts during the flight. In this paper, the elastic cable is modeled as a finite number of $N$-link springs where the mass of each link is lumped at the associated joint. Figure 2 depicts the towed cable system in the simulation, where the mothership ( $\mathbf{p}_{m}$ ) is modeled as a point mass, the cable is modeled as a finite number of point mass nodes ( $\mathbf{p}_{1}$ to $\mathbf{p}_{N}$ ) connected by springs, and the drogue $\left(\mathbf{p}_{N}\right)$ is modeled as the last joint of the cable. Even though the equations of motion of the cable-drogue system given in this section are similar to those in [20] and the equations of applied forces are similar to those in [12], they are repeated here for completeness.

\section{A. Equations of motion of the cable-drogue system}

In this paper, cable-drogue dynamics are expressed in the inertial North-East-Down (NED) coordinate frame. The cable is discretized into $N$ rigid springs with masses lumped at each joint. According to Newton's second law, the acceleration of the $j^{\text {th }}, j=1,2, \cdots, N$, joint $\mathbf{a}_{j} \in \mathbb{R}^{3}$ is given by

$$
\mathbf{a}_{j}=\frac{\mathbf{F}_{j}}{m_{j}},
$$

where $\mathbf{F}_{j} \in \mathbb{R}^{3}$ represents applied forces exerted on the $j^{\text {th }}$ joint, and $m_{j}$ is the mass of the $j^{\text {th }}$ link. 


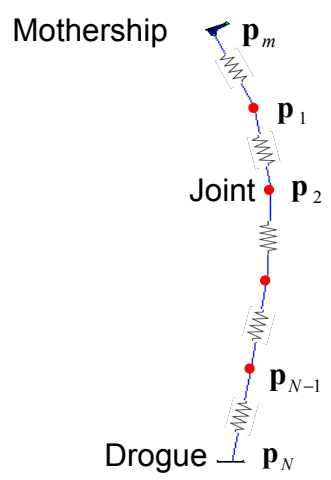

Fig. 2. The mothership-cable-drogue system in the simulation.

In this paper, the last cable joint and the drogue are combined together as a single mass point and the mass of the last cable link is lumped with the drogue. Assuming that the mass of the cable $m_{c}$ is uniformly distributed, lumped masses of cable joints become

$$
\begin{aligned}
m_{j} & =\frac{m_{c}}{N}, j=1,2, \cdots, N-1, \\
m_{N} & =\frac{m_{c}}{N}+m_{d r}
\end{aligned}
$$

where $m_{d r}$ is the mass of the drogue.

Letting $\mathbf{p}_{j} \in \mathbb{R}^{3}, j=1,2, \cdots, N$, be the position of the $j^{\text {th }}$ joint in the NED frame (drogue as the last joint), $\mathbf{w}_{c} \in \mathbb{R}^{3}$ be the estimated wind speed, $\mathbf{v}_{j}$ be the velocity vector of the $j^{\text {th }}$ cable joint relative to the wind frame, the equations of motion of the cable-drogue system can be written as

$$
\begin{aligned}
\dot{\mathbf{p}}_{j} & =\mathbf{v}_{j}+\mathbf{w}_{c}, \\
\ddot{\mathbf{p}}_{j} & =\dot{\mathbf{v}}_{j}=\mathbf{a}_{j} .
\end{aligned}
$$

It should be noted that in this paper, the wind is modeled as a combination of two components: an estimated portion $\left(\mathbf{w}_{c}\right)$ treated as a time-varying parameter and an unmeasured gust. The wind estimation algorithm is assumed to be sufficiently accurate to measure the dominant component of the instantaneous wind speed. The wind gust is treated as an unmeasured disturbance which is much smaller than $\mathbf{w}_{c}$.

\section{B. Applied forces}

Assuming that applied forces on the $j^{\text {th }}$ joint $\mathbf{F}_{j}$ include the gravity $\mathbf{G}_{j} \in \mathbb{R}^{3}$, aerodynamic forces $\mathbf{F}_{j}^{\text {aero }} \in \mathbb{R}^{3}$, and the cable tension $\mathbf{F}_{j}^{t} \in \mathbb{R}^{3}$, then

$$
\mathbf{F}_{j}=\mathbf{G}_{j}+\mathbf{F}_{j}^{a e r o}+\mathbf{F}_{j}^{t}
$$


Letting $\mathbf{e}_{3} \triangleq(0,0,1)^{T}$ be the unit vector in the down direction in NED coordinates, and $g_{c}$ be the gravitational force exerted on a unit mass at Earth sea level, the gravity force exerted on the $j^{\text {th }}$ joint is given by

$$
\begin{aligned}
\mathbf{G}_{j} & =m_{j} g_{c} \mathbf{e}_{3}, j=1,2, \cdots, N-1, \\
\mathbf{G}_{N} & =\left(m_{N}+m_{d r}\right) g_{c} \mathbf{e}_{3} .
\end{aligned}
$$

Assuming that aerodynamic forces on each joint are negligible and that each rigid link is a cylinder, the cross-flow principle $[12,14,21]$ can be used to calculate aerodynamic forces acting on cable links.

The position of the towing vehicle (mothership), $\mathbf{p}_{m} \in \mathbb{R}^{3}$, is considered as the position of the $0^{\text {th }}$ joint of the cable, $\mathbf{p}_{0} \in \mathbb{R}^{3}$. Letting $\mathbf{q}_{j} \triangleq \mathbf{p}_{j-1}-\mathbf{p}_{j}$ be the vector between the $(j-1)^{\text {th }}$ and the $j^{\text {th }}$ joints, the velocity vector of the $j^{\text {th }}$ cable link relative to the wind frame, $\mathbf{v}_{j}^{s}$, can be approximated from the motion of adjacent joints as

$$
\mathbf{v}_{j}^{s}=\frac{\left(\dot{\mathbf{p}}_{j-1}-\mathbf{w}_{c}\right)+\left(\dot{\mathbf{p}}_{j}-\mathbf{w}_{c}\right)}{2} .
$$

Then, the angle of attack of the $j^{\text {th }}$ link, $\alpha_{j}$, can be computed as

$$
\alpha_{j}=\cos ^{-1}\left(\frac{\mathbf{q}_{j}^{T} \mathbf{v}_{j}^{s}}{\left\|\mathbf{q}_{j}\right\|\left\|\mathbf{v}_{j}^{s}\right\|}\right) .
$$

Letting $M_{p_{j}}$ and $M_{n_{j}}$ be Mach numbers parallel and normal to the $j^{\text {th }}$ link, respectively, the drag and lift coefficients of the $j^{\text {th }}$ link are given by $[12,14,21]$

$$
\begin{aligned}
C_{D_{j}} & =C_{f_{j}}+C_{n_{j}} \sin ^{3} \alpha_{j}, \\
C_{L_{j}} & =C_{n_{j}} \sin ^{2} \alpha_{j} \cos \alpha_{j},
\end{aligned}
$$

where the skin-friction and cross-flow drag coefficients are given by $[12,14,21]$

$$
\begin{aligned}
C_{f_{j}} & =0.038-0.0425 M_{p_{j}}, \\
C_{n_{j}} & =1.17+M_{n_{j}} / 40-M_{n_{j}}^{2} / 4+5 M_{n_{j}}^{3} / 8 .
\end{aligned}
$$

Directions of the aerodynamic lift and drag forces of each link can be determined using the following unit vectors $[12,21]$

$$
\begin{aligned}
\mathbf{e}_{D_{j}} & =-\frac{\mathbf{v}_{j}^{s}}{\left\|\mathbf{v}_{j}^{s}\right\|}, \\
\mathbf{e}_{L_{j}} & =-\frac{\left(\mathbf{v}_{j}^{s} \times \mathbf{q}_{j}\right) \times \mathbf{v}_{j}^{s}}{\left\|\left(\mathbf{v}_{j}^{s} \times \mathbf{q}_{j}\right) \times \mathbf{v}_{j}^{s}\right\|},
\end{aligned}
$$

where $\times$ denotes the cross product of two vectors. Letting $\rho$ be the atmospheric density and $d$ be the cable diameter, the drag and lift forces acting on the $j^{\text {th }}$ link are given by

$$
\begin{aligned}
\mathbf{D}_{j} & =\frac{1}{2} \rho C_{D_{j}}\left\|\mathbf{q}_{j}\right\| d\left\|\mathbf{v}_{j}^{s}\right\|^{2} \mathbf{e}_{D_{j}} \\
\mathbf{L}_{j} & =\frac{1}{2} \rho C_{L_{j}}\left\|\mathbf{q}_{j}\right\| d\left\|\mathbf{v}_{j}^{s}\right\|^{2} \mathbf{e}_{L_{j}}
\end{aligned}
$$


Assuming that these lift and drag vectors are constant over each cable link, and that the aerodynamic forces are lumped and centered at the midpoint of each link, aerodynamic forces exerted on the $j^{\text {th }}$ joint are given by

$$
\mathbf{F}_{j}^{a e r o}=\frac{1}{2}\left[\left(\mathbf{D}_{j}+\mathbf{D}_{j+1}\right)+\left(\mathbf{L}_{j}+\mathbf{L}_{j+1}\right)\right], j=1,2, \cdots, N-1 .
$$

Aerodynamic forces on the last joint (drogue) are associated with both the last cable link and the drogue. Similarly, the aerodynamic lift and drag forces on the drogue can be calculated as

$$
\begin{aligned}
\mathbf{D}_{d r} & =-\frac{1}{2} \rho C_{D_{d r}} S_{d r}\left\|\mathbf{v}_{d r}^{s}\right\| \mathbf{v}_{d r}^{s} \\
\mathbf{L}_{d r} & =\frac{1}{2} \rho C_{L_{d r}} S_{d r}\left\|\mathbf{v}_{d r}^{s}\right\|^{2} \mathbf{e}_{L_{d r}} \\
\mathbf{e}_{L_{d r}} & =-\frac{\left(\mathbf{v}_{d r}^{s} \times \mathbf{e}_{3}\right) \times \mathbf{v}_{d r}^{s}}{\left\|\left(\mathbf{v}_{d r}^{s} \times \mathbf{e}_{3}\right) \times \mathbf{v}_{d r}^{s}\right\|}
\end{aligned}
$$

where $C_{D_{d r}}$ and $C_{L_{d r}}$ are the aerodynamic drag and lift coefficients of the drogue, respectively, $S_{d r}$ is the planform wing area of the drogue, $\mathbf{v}_{d r}^{s}$ is the velocity vector of the drogue relative to the wind frame, $\mathbf{e}_{L_{d r}}$ is the unit vector representing the direction of the aerodynamic lift force on the drogue. Then, the aerodynamic force exerted on the last joint of the cable is given by

$$
\mathbf{F}_{N}^{a e r o}=\frac{1}{2}\left(\mathbf{D}_{N}+\mathbf{L}_{N}\right)+\mathbf{D}_{d r}+\mathbf{L}_{d r}
$$

Tension forces on each link exist only when the link is stretched, and are always along the cable segment. Letting $\mu_{0} \triangleq L_{0} / N$ be the original length of each link, where $L_{0}$ is the original length of the entire cable, then according to Hooke's law, the tension force exerted on the $j^{\text {th }}$ joint by the $(j-1)^{\text {th }}$ joint, $\mathbf{T}_{j} \in \mathbb{R}^{3}$, is given by

$$
\mathbf{T}_{j}=\frac{E A}{2 \ell_{0}}\left(\|\| \mathbf{q}_{j}\left\|-\mu_{0}\right\|+\left\|\mathbf{q}_{j}\right\|-\mu_{0}\right) \frac{\mathbf{p}_{j-1}-\mathbf{p}_{j}}{\left\|\mathbf{p}_{j-1}-\mathbf{p}_{j}\right\|},
$$

where $E$ is the Young's modulus and $A$ is the cross-sectional area of the cable. Thus total tension forces for each joint are given by

$$
\begin{aligned}
\mathbf{F}_{j}^{t} & =\mathbf{T}_{j}-\mathbf{T}_{j+1}, j=1,2, \cdots, N-1, \\
\mathbf{F}_{N}^{t} & =\mathbf{T}_{N} .
\end{aligned}
$$

\section{FRAMEWORK FOR PARAMETER ESTIMATION}

Although the equations of motion for the aircraft, towed cable, and drogue were developed from fundamentals, there remained inaccuracies in the model. These inaccuracies result from uncertain estimates of particular parameters, simplifications from model assumptions to make the solution computationally tractable, asynchronous measurements due to hardware limitations, and unmeasured disturbances, among others. When the model was employed to optimize the path trajectory of the drogue with cross-wind, discrepancies of altitude between the measured and model values of the drogue were observed to be up to $30 \mathrm{~m}$. This large discrepancy motivated the current work to correct and adapt the model. 


\section{A. Parameter estimation using Kalman filters}

Traditional estimation techniques for nonlinear systems such as the extended Kalman filter (EKF) and unscented Kalman filter (UKF) were first employed to estimate parameters, e.g., the cable length and the aerodynamic drag coefficient of the drogue, in towed cable systems. For the EKF, due to the complexity of the equations of motion presented in the previous section, it is nontrivial to obtain the closed form of the Jacobian matrix. Thus, a numerical method is utilized to calculate the Jacobian matrix at each cycle. To simplify the computation complexity, a 2-link cable is used to establish dynamic equations of the system. Although using simulation data with $0.02 \mathrm{~s}$ sampling rate generates reasonable estimates, the estimation results obtained from using both the EKF and UKF based on flight test data with $1 \mathrm{~s}$ sampling rate diverged. Other than the reasons stated in the subsequent analysis in Section IV-B, the diverged estimates also result from flight test data of a relatively large sampling time (1 s) and asynchronous mothership and drogue measurements. This causes the acceleration of the joint (with a relatively small weight) to quickly reach an infinite value. Therefore, an alternative technique is pursued to overcome the deficiencies of the EKF and UKF. The next section describes an optimization based moving horizon estimation.

\section{B. Parameter estimation using moving horizon estimation}

While state and parameter estimation for nonlinear systems is not new research, certain aspects of the approach are novel. One new contribution is the formulation of the estimation objective function that optimally rejects measurement noise without the requirement for state error and noise covariance information. The inverse of the state error and noise covariance matrices are typically used to define the weighting factors on the balance between how much of the new state estimate is derived from the measurements versus the prior state estimate. Estimating these covariance matrices can be challenging for large-scale systems or when there is no structure to the covariance information thereby resulting in dense matrices. The methods proposed in this section require only an estimate of accuracy of each sensor. One drawback of this method is that additional equations and variables must be added to formulate the objective function, leading to additional computational expense.

An area of concern was whether these estimation algorithms can be used to optimally estimate states and parameters in real-time. Offline studies suggested that the computational expense and communication lag would be too great to match the frequency of the data stream (up to $10 \mathrm{~Hz}$ ) but that it could be run at a time-scale faster than the dominant time constant of the towed cable system $(0.5-1.0 \mathrm{~Hz})$. The method in this research is to compensate for the effect of additional problem size and computational expense by using efficient nonlinear programming (NLP) solvers and sparse matrix operations. The objective is to achieve real-time state and parameter estimation using a nonlinear dynamic model, asynchronous data sources, and noisy data for this towed cable system.

To apply efficient NLP solvers, the equations of motion were posed in a standard form of differential and algebraic equations (DAE) in open-equation format and a nonlinear objective function as

$$
\min _{\mathbf{u}, \mathbf{z}} \Phi(\mathbf{x}, \mathbf{y}, \mathbf{z}, \mathbf{u})
$$




$$
\begin{aligned}
\text { s.t. } \mathbf{0} & =\mathbf{f}\left(\frac{\partial \mathbf{x}}{\partial t}, \mathbf{x}, \mathbf{y}, \mathbf{z}, \mathbf{u}\right) \\
\mathbf{0} & =\mathbf{g}_{e}(\mathbf{x}, \mathbf{y}, \mathbf{z}, \mathbf{u}) \\
\mathbf{0} & \leq \mathbf{h}(\mathbf{x}, \mathbf{y}, \mathbf{z}, \mathbf{u})
\end{aligned}
$$

where $\Phi$ is a multi-tiered objective function of any continuously differentiable form that is minimized subject to differential $\mathbf{f}$, algebraic $\mathbf{g}_{e}$, and inequality $\mathbf{h}$ constraints, $\mathbf{y}$ represents measured values, $\mathbf{u}$ represents exogenous inputs at the sampling points, $\mathbf{g}_{e}$ represents equation residuals that relate the states to measured outputs, and $\mathbf{h}$ represents inequality constraints. The solution of (19) is determined by the initial state $\mathbf{x}_{0}$ (e.g. initial position and velocity of mothership and drogue) and a sequence of exogenous inputs. The exogenous inputs may include parameters (e.g. drag coefficient of the drogue, the Young's modulus of the cable), measured disturbances (e.g. wind speed), past control inputs (e.g. mothership control efforts), or other independently specified values. Some or all of the state values $\mathbf{x}$ may also be measured values $\mathbf{y}$ that may be used to update both parameters $\mathbf{u}$ and initial conditions $\mathbf{x}_{0}$. Integer variables $\mathbf{z}$ are also included to represent states or inputs that can only take integer values. The equations for $\mathbf{x}$ and $\mathbf{y}$ are solved at specific points over the time horizon to make the problem tractable for numerical solution. This solution technique is used in the APMonitor Modeling Language [44] as a toolbox for MATLAB ${ }^{\circledR}$ or Python.

For this specific problem, the equations of motion that relate position, velocity (e.g. Equation (7)), and acceleration (e.g. Equation (1)) are converted to first-order differential equations that are fit into the form of (19) by including them as the (19b) set of equations. Furthermore, Equations (2)-(6) and (8)-(18) are algebraic equations that are lumped into the set with (19c) as equality constraints. The inequality constraints as denoted by (19d) are upper constraints on certain variables such as cable tension and the slack variables used to define the error model as seen later in $(20 \mathrm{e})-(20 \mathrm{i})$.

Internal to APMonitor, the DAE model is converted to a mixed-integer nonlinear programming (MINLP) form by approximating the derivative values $\frac{\partial \mathbf{x}}{\partial t}$ through a direct transcription approach called orthogonal collocation on finite elements [45]. The MINLP problems are solved by large-scale MINLP solvers such as APOPT [46] or Bonmin/IPOPT [47]. Additional details on the transformation from DAE to MINLP are given in [33, 48].

A strategy is then developed to produce optimal estimates of the specified parameters of the towed cable system given a real-time stream of measurements and subject to the system constraints and equations of motion. In the literature, when formulating the optimal estimates for system update, the nonlinear kinematic or dynamic models of the system are typically used, and the objective functions are usually based on squared error or $\ell_{2}$-norm form (e.g. Kalman filtering). To improve the accuracy of the estimation, the nonlinear dynamic equations of the system are given and a novel form of the objective function is used to formulate the MHE problem. This novel form has a dead-band around the current measurements in order to reject noise and an $\ell_{1}$-norm penalty outside of the noise dead-band. The implementation of the objective function in (19) is a formulation that can be solved by numerical techniques for large-scale models. Slack variables are used to implement inequality constraints that emulate an absolute value operation. The resulting combination of slack variables and inequalities is continuously 
differentiable as required by NLP solvers.

The $\ell_{1}$-norm form serves a number of purposes including rejection of data outliers that would otherwise shift the state and parameter estimates from true values. The outliers are not identified and eliminated by heuristic or bounding methods but are naturally discounted during the solution of the optimization problem. This discounting occurs because the optimizer is minimizing a collection of measurements over a time horizon. If the outliers are infrequent compared with the total number of measurements, the optimal solution will be obtained by minimizing a majority of the measurement deviations from the predicted model values. Objective functions with an $\ell_{2}$-norm are disproportionately affected by outliers because the error is squared, leading to a greater contribution by large terms. The specific equations incorporating dead-bands are given by

$$
\begin{aligned}
\min _{\mathbf{u}, \mathbf{z}} \Phi & =\mathbf{w}_{m}^{T}\left(\mathbf{e}_{U}-\mathbf{e}_{L}\right)+\mathbf{w}_{p}^{T}\left(\mathbf{c}_{U}+\mathbf{c}_{L}\right) \\
\text { s.t. } \mathbf{0} & =\mathbf{f}\left(\frac{\partial \mathbf{x}}{\partial t}, \mathbf{x}, \mathbf{y}, \mathbf{z}, \mathbf{u}\right) \\
\mathbf{0} & =\mathbf{g}_{e}(\mathbf{x}, \mathbf{y}, \mathbf{z}, \mathbf{u}) \\
\mathbf{0} & \leq \mathbf{h}(\mathbf{x}, \mathbf{y}, \mathbf{z}, \mathbf{u}) \\
\mathbf{e}_{U} & \geq \mathbf{y}-\mathbf{y}_{U} \\
\mathbf{e}_{L} & \geq \mathbf{y}_{L}-\mathbf{y} \\
\mathbf{c}_{U} & \geq \mathbf{y}-\hat{\mathbf{y}} \\
\mathbf{c}_{L} & \geq \hat{\mathbf{y}}-\mathbf{y} \\
\mathbf{0} & \leq \mathbf{e}_{U}, \mathbf{e}_{L}, \mathbf{c}_{U}, \mathbf{c}_{L}
\end{aligned}
$$

where $\hat{\mathbf{y}}$ is the prior model values at sampling times $\left(\hat{\mathbf{y}}_{0}, \ldots, \hat{\mathbf{y}}_{n}\right)^{T}, \mathbf{w}_{m}$ is the weight on the model values outside a measurement dead-band, $\mathbf{w}_{p}$ is the weight to penalize deviation from the prior solution, $\mathbf{e}_{U}$ and $\mathbf{e}_{L}$ is the slack variables to penalize model values above and below the measurement dead-band, and $\mathbf{c}_{U}$ and $\mathbf{c}_{L}$ are the slack variables to penalize model value changes above and below the prior model estimates.

Equations (20e)-(20i) deserve additional explanation because they constitute a unique contribution from this work. These inequality constraints define a dead-band around the measured values to allow the model value to be deemed acceptable if it falls within that range. Another way of explaining this form is that if the model value is between the upper $\left(\mathbf{y}_{U}\right)$ and lower $\left(\mathbf{y}_{L}\right)$ limits, there is no penalty in the objective function at that time point. If the model value is outside of this range $\left(\mathbf{y}_{L}\right.$ to $\left.\mathbf{y}_{U}\right)$ to then there is a linear contribution that is weighted by $\mathbf{w}_{m}$. The variables $\mathbf{e}_{U}$ and $\mathbf{e}_{L}$ are always positive and are the upper and lower errors, respectively.

Similarly, $\mathbf{c}_{U}$ and $\mathbf{c}_{L}$ are defined to penalize deviation from the prior state estimates. This penalty is a "forgetting factor" that approximates the infinite horizon solution with a finite time horizon. Any deviation from the prior state estimate $\mathbf{y}_{m}$ is multiplied by the weighting factor $\mathbf{w}_{p}$ and added to the objective function in (20a). The values of $\mathbf{c}_{U}$ and $\mathbf{c}_{L}$ are always positive and represent the positive deviation and negative deviation from the prior state estimate, respectively. 
The objective form in (20) accomplishes a number of desirable characteristics. First the parameters are only adjusted if the measured value is more than half of the dead-band away from the model value. Otherwise, the parameters that affect the model predictions are not adjusted because the measurement lies within the region of dead-band. Second, multiple objectives are solved with a single optimization. For example, in the case of redundant measurements, explicit prioritization can be enforced by increasing the objective function gradient with weighting factors to selectively track measurements. Secondary measurements are only utilized when the primary measurements become unavailable or are infrequently updated. Third, weightings in the objective function on prior states are an approximation to the infinite horizon solution that retains some of the prior information without an excessive horizon length or computational expense. These qualities are particularly desirable for estimation of unmanned aircraft systems that must be robust to measurement drift, failure, outliers, infrequent data, delayed data, and utilize redundant measurement devices seamlessly.

In this paper, the model consists of the dynamic equations that describe the motion of the mothership, cable, and drogue. The available measurements that are updated at frequent intervals include GPS coordinates for both the mothership and drogue, wind velocity estimates in the two horizontal directions at the altitude of the mothership, and the airspeed of the mothership. The difference between the model and measured values is minimized subject to the model equations with adjustable parameters like the cable length due to tension, the number of cable links (integer parameter), drag coefficient of the drogue, wind velocity in the vertical direction, and the Young's modulus of the cable.

\section{EXPERIMENTAL RESULTS}

In this section, the MHE based estimation algorithm proposed in the previous section is applied to an aerially towed cable system as described in Section II. Experiments are designed to test the effectiveness of the algorithm as well as to investigate its real-time practicability. The applied cable length, a significant parameter that strongly dictates system dynamics in simulation, was selected as a nominal parameter to be estimated. Although the cable length is a parameter that can be readily measured before an experiment, the fishing-line cable used in the current experiment has been observed to stretch and contract in previous flight tests. Also, an approximated mathematical model with roughly-measured parameters like the Young's modulus may not be accurate enough to fully capture system dynamics like a cable stretching. Therefore, the applied value of the original cable length in a mathematical model should be further validated in order for the approximated mathematical representation to better reconcile the simulated system dynamics with the actual one. This enables a more simplified model to potentially capture more realistic flight dynamics. The hardware platform and experimental results are described in the rest of the section.

\section{A. Hardware platform}

The hardware system used in the flight test, as shown in Figure 3, consists of three key components: a towing UAV (mothership), a passive drogue without controllable surfaces and connected to the mothership by a nylon cable, and a ground station with associated communication devices. Some key parameters of the system are given 


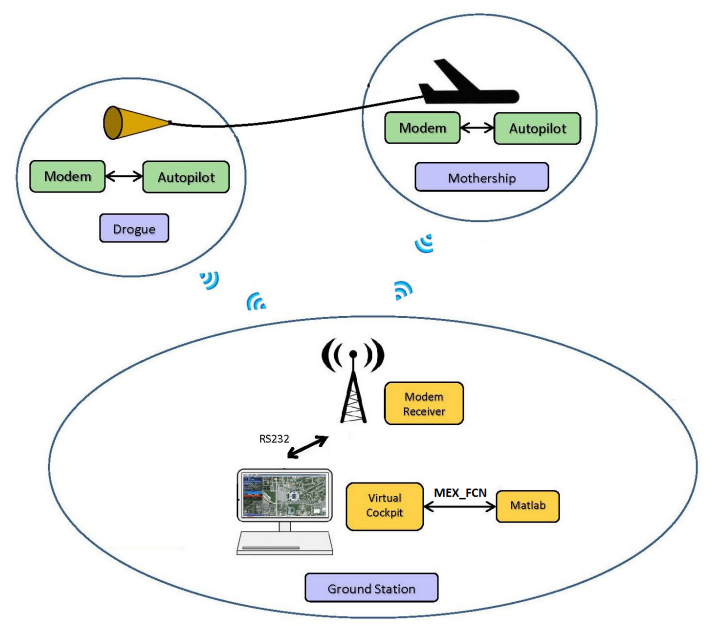

Fig. 3. Aerially towed-cable system hardware.

in Table I and were obtained from cruise flight conditions in a wind tunnel, weight scales, and length measurement devices.

TABLE I

SYSTEM PARAMETERS IN FLIGHT TEST.

\begin{tabular}{|c|c||c|c||c|c|}
\hline \multicolumn{2}{|c||}{ Mothership } & \multicolumn{2}{c||}{ Drogue } & \multicolumn{2}{c|}{ Cable } \\
\hline Mass $(\mathrm{kg})$ & 1.76 & $m_{d r}(\mathrm{~kg})$ & 0.32 & $m_{c}(\mathrm{~kg})$ & 0.02 \\
\hline$C_{L}$ & 0.28 & $C_{L}$ & 0.01 & $\ell_{0}(\mathrm{~m})$ & 85 \\
\hline$C_{D}$ & 0.06 & $C_{D}$ & 0.42 & $E(\mathrm{GPa})$ & 1.9 \\
\hline Wing area $\left(\mathrm{m}^{2}\right)$ & 0.307 & Diameter $(\mathrm{m})$ & 0.3 & $d(\mathrm{~mm})$ & 0.46 \\
\hline Wing span $(\mathrm{m})$ & 1.4 & & & & \\
\hline
\end{tabular}

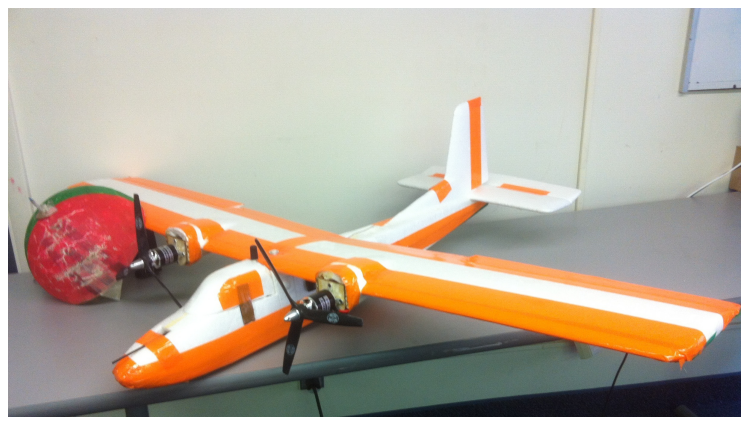

Fig. 4. Mothership and drogue (hemisphere resting on right wing).

1) Mothership and drogue: As shown in Figure 4, the mothership is a Multiplex Twin Star airframe model equipped with a Kestrel 2 autopilot and a modem used to communicate with the ground station. The mothership is 
powered using two $5000 \mathrm{mAh}$ lithium polymer batteries connected to twin $910 \mathrm{~W}$ Turnigy motors typically using about $500 \mathrm{~W}$ each. The mothership has a maximum flight time of $30 \mathrm{~min}$ with the cable and drogue attached. The applied hemisphere drogue is made of reinforced plastic, and is also equipped with a Kestrel 2 autopilot and a modem used to report its position and velocity to the ground station.

2) Ground station: The ground station consists of a desktop computer with Intel i5 processor running at $3.1 \mathrm{GHz}$ with 8 GB RAM, a modem receiver and a remote controller. The flight control and monitoring software Virtual Cockpit is installed on the computer and connected to the modem receiver for communicating with the autopilots on both mothership and drogue with a bandwidth at $115.2 \mathrm{~KB} / \mathrm{s}$. Estimation and control strategies are implemented in MATLAB ${ }^{\circledR}$ which is installed in the computer and communicates with Virtual Cockpit using a MATLAB ${ }^{\circledR}$ Executable (MEX) function with a communication rate at $5 \mathrm{~Hz}$.

\section{B. Real-time parameter estimation}

In flight tests, the mothership was commanded to follow a loiter of $250 \mathrm{~m}$ radius and a constant altitude of $150 \mathrm{~m}$ with the airspeed commanded at $14 \mathrm{~m} / \mathrm{s}$. The system trajectory and estimated wind speed on the mothership level are shown in Figure 5. The top-down view of the system trajectory is presented in Figure 5 (a), where the drogue orbit was essentially overlaid on the mothership orbit because a mothership orbit with relatively large radius (150-250 m) and a relatively short cable $(70-85 \mathrm{~m})$ were used. This experimental system allowed validation of the model even though the velocity and dimensions of an actual MAV capture system would likely be much larger. The east-altitude view of the system trajectory is shown in Figure 5 (b). It can be seen that the mothership trajectory was essentially on a horizontal orbit, while the resulting drogue orbit had an oscillation of approximately $20 \mathrm{~m}$ in the altitude due to the wind. Figure 5 (c) shows the wind speed estimation in the north and east directions, respectively. The magnitude of the wind was approximately $2 \mathrm{~m} / \mathrm{s}$.

The mothership position, airspeed, and estimated wind speed at the mothership altitude level in the flight test are selected as the inputs of the simulation. Parameters like the applied cable length in the mathematical model are automatically manipulated by the solver to minimize the difference between the drogue position calculated using the mathematical model and the measured position in the flight test. In the experiment, the estimated wind speed at the drogue altitude level was assumed to be the same as that at the mothership altitude level. The number of cable links were selected as two to decrease the model complexity [22]. The communication rate between Virtual Cockpit and MATLAB ${ }^{\circledR}$ is $5 \mathrm{~Hz}$, i.e., the sample step size between each measurement is $0.2 \mathrm{~s}$. The optimization solver was selected as APOPT [46]. Before the flight test, off-line simulations were conducted to test whether the computation time of each cycle is smaller than the communication rate $(5 \mathrm{~Hz})$ by using the ground station computer introduced in IV-A2. The maximal computation time in each cycle for 100 runs is less than $0.08 \mathrm{~s}$, which meets the requirement for real-time estimation. 


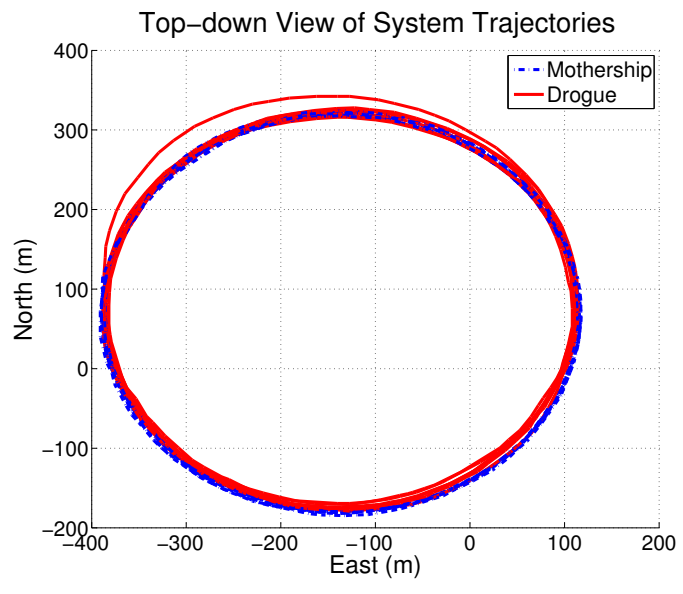

(a) Top-down view.

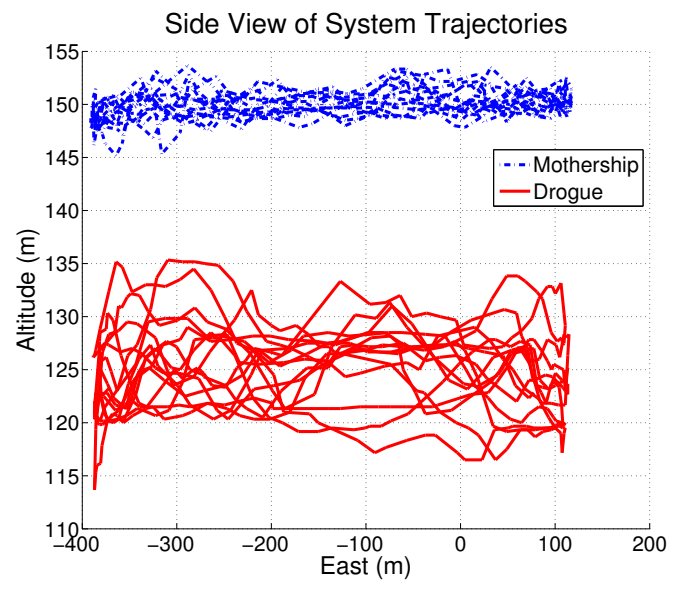

(b) Side (east-altitude) view.
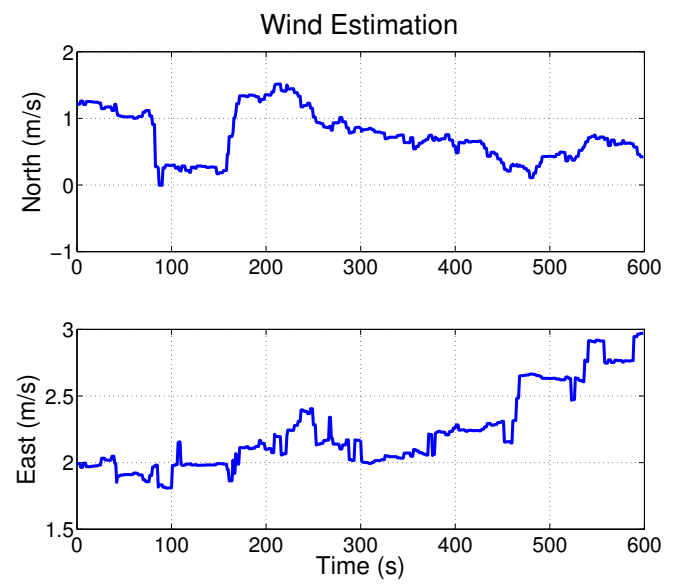

(c) Estimated wind speed.

Fig. 5. System trajectories and wind estimation.
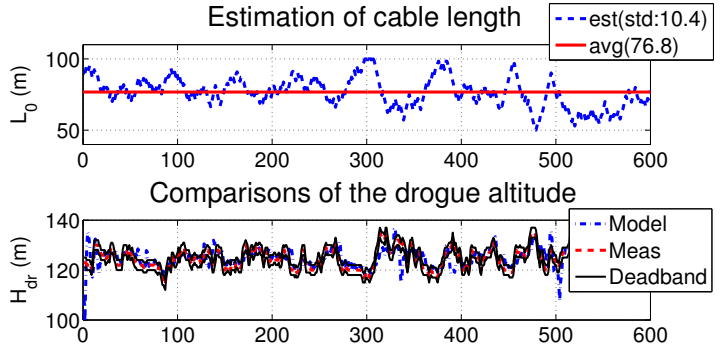

Drogue altitude difference of meas and model values

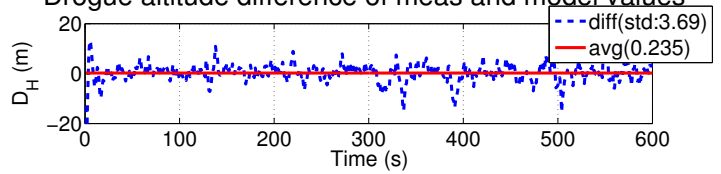

Fig. 6. Real-time estimation results. 
Figure 6 shows real-time experimental results of the applied cable length. The upper subfigure of Figure 6 shows the evolution of the estimation throughout $600 \mathrm{~s}$ of flight time. The average of the estimates is $76.8 \mathrm{~m}$ and the estimation oscillated between $50 \mathrm{~m}$ and $100 \mathrm{~m}$ with the standard deviation as $10.4 \mathrm{~m}$. Measured values (dash line) and model values (dot-dash line) of the drogue altitude are overlaid in the middle subfigure of Figure 6 . The deadband (solid line) was selected as $\pm 2 \mathrm{~m}$ of the measured value. The model value is essentially in the dead-band except for several large deviations. The difference between the measured value and model value is shown in the bottom subfigure of Figure 6. The standard deviation of the error is $3.69 \mathrm{~m}$ and the average error is $0.235 \mathrm{~m}$.

Relatively large oscillations of the estimates result from several sources of uncertainty. First, the autopilot uses measurements of ground speed (obtained from GPS), course angle (obtained from GPS), and airspeed (obtained from the pitot tube) to estimate the instantaneous direction and magnitude of the wind speed. However, due to gusts and the sensitivity of sensors, an instantaneous estimate of the wind speed is noisy. It is also assumed that the estimated wind speeds on the mothership and drogue levels are identical, and the wind speed in the vertical direction is zero. Second, the hardware platform is a multi-agent system where the mothership and drogue are equipped with separate autopilots. The system complexity increases the communication delay between vehicles and the ground station. Therefore, measurements collected from the mothership and drogue are not well synchronized. Also, the GPS sensors on both vehicles have a drifting variance of approximate $3 \mathrm{~m}$ in the altitude measurement. Third, the configuration of MHE uses $1 \mathrm{~s}$ time horizon, which may not be sufficient to produce smooth estimates. Last but not least, as mentioned before, the cable stretching can also result in large oscillations of the estimates.

Due to limited experimental time, the applied cable length was the only parameter estimated during real-time flight conditions. The following section addresses common challenges to other nonlinear dynamic systems where parameters range from fully-observable to unobservable. Although fully observable, some unknown parameters are constant or vary stochastically. The following analysis does not consider all possible combinations of parameters but does give some insight on which parameters to estimate during future real-time flight tests.

\section{OfF-Line ESTIMATION RESUlts}

In this section, comprehensive off-line estimations in simulation are conducted to further investigate the effectiveness of the proposed approach in estimating parameters of interest. Estimation of single parameters, such as the drag coefficient of the drogue, the Young's modulus and the vertical component of the wind speed, is performed. Different horizon window size (1, 5, and 10 seconds) and different number of cable links (2 to 9) are applied to analyze the influence of mathematical model complexity on solution accuracy. Furthermore, the ability to simultaneously estimate multiple parameters using the proposed approach is tested. In this section, the raw flight test data are trimmed and selected as 600 data sets with a $1 \mathrm{~s}$ interval, which represents a $10 \mathrm{~min}$ flight period that comprises multiple orbits of the system. 


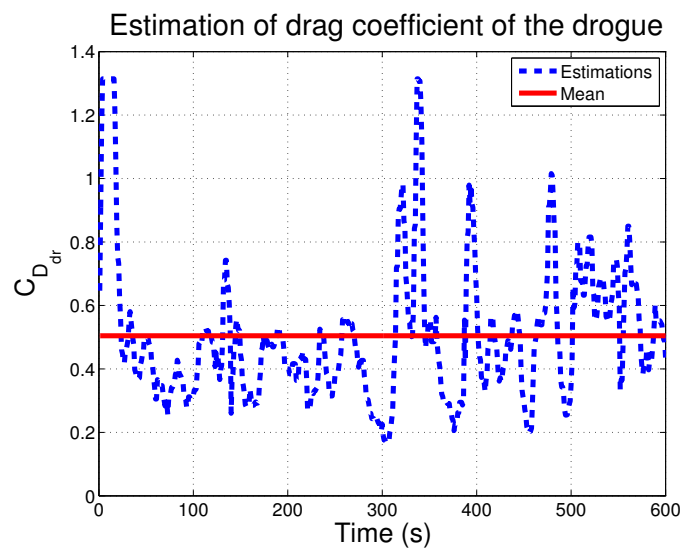

Fig. 7. The estimation of $C_{D_{d r}}$ using a horizon time of 10 seconds.

First, a 2-link cable model and horizon times of 1 and 5 seconds are used to perform the estimation of the drag coefficient of the drogue $\left(C_{D_{d r}}\right)$. However, the resulting estimates oscillate with large amplitude and frequency and do not provide consistent results. The corresponding coefficients of variation (the ratio of the standard deviation over the mean) are 0.72 and 0.57 , respectively. Figure 7 shows the estimation of $C_{D_{d r}}$ using a 2-link cable model and a horizon time of 10 seconds with a coefficient of variation of 0.48 . Using a larger horizon time, a solver is able to capture a broader history of system states and is able to better smooth out unwanted variation. However, as a trade-off, computational (CPU) times corresponding to horizon times of 1,5 , and 10 seconds are 0.04 , 0.1 , and 0.18 seconds, respectively. Figure 7 also shows that the average of the estimates is approximately 0.5 , which is a reasonable value for a nominal $C_{D_{d r}}$.

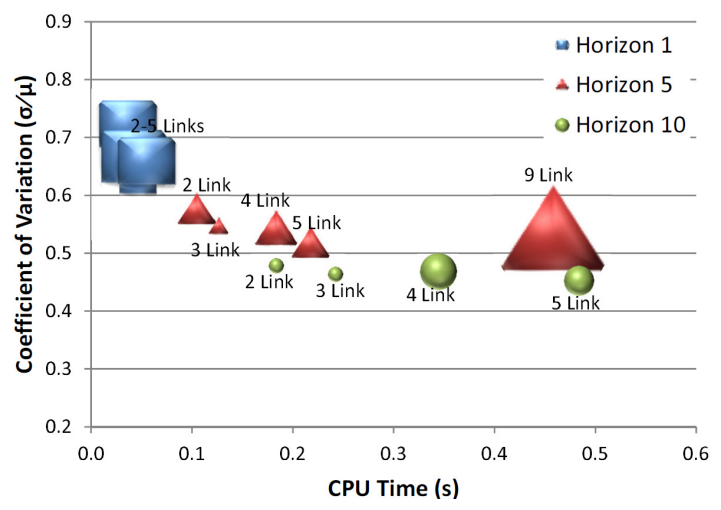

Fig. 8. Results of $C_{D_{d r}}$ estimation using different numbers of cable links and horizon times.

Figure 8 shows an analysis of the tradeoff between the coefficient of variation and the CPU time using different number of cable links and different horizon time. Each object represents a specific horizon time and the number of 
cable links. Different markers, square, triangle and circle, represent horizon times 1, 5, and 10 seconds, respectively. The number of cable links is also labeled next to each marker. The center of each marker is the intersection of the resulting coefficient of variation and the CPU time. The marker size represents the amount of failed solutions, primarily caused by the inability of the solver to find a solution within a specified maximum number (200) of iterations. Another possible cause for failure is that the hard constraints may lead to an infeasible set that makes the solver terminate with an error message saying no solution. It can be seen from Figure 8 that solutions using a 1 -second time horizon (squares) require smaller (less than $0.1 \mathrm{~s}$ ) CPU times but have a relatively large coefficient of variation (approximately 0.7). However, an increase in the number of cable links does not result in an obvious increase of both the CPU time and the number of failed solutions. When the horizon time of 5 second is used in simulation, estimates (triangles) have a smaller coefficient of variation (between 0.5 to 0.6). As the number of cable links increases from 2 to 5 , the CPU time slightly increases from approximately 0.1 to 0.2 second, while the number of failed solutions does not have an obvious variation. When the horizon time increases to 10 second, the coefficient of variation stays between 0.4 to 0.5 . However, as the number of cable links increases from 2 to 5 , the CPU time increases from approximately 0.2 to 0.5 , respectively, while the number of failed solutions decreases when using 2-link and 3-link cable models and increases again when using 4-link and 5-link cable models. A simulation is also conducted using a 9-link cable model and a 5-second horizon time. The purpose is to see whether an increase in the number of cable links would result in a decrease in the coefficient of variation and the number of failures. Unfortunately, the resulting CPU time increases to approximately $0.45 \mathrm{~s}$ and the number of failures increases abruptly, as shown in Figure 8.

\section{B. Determination of an optimal configuration}

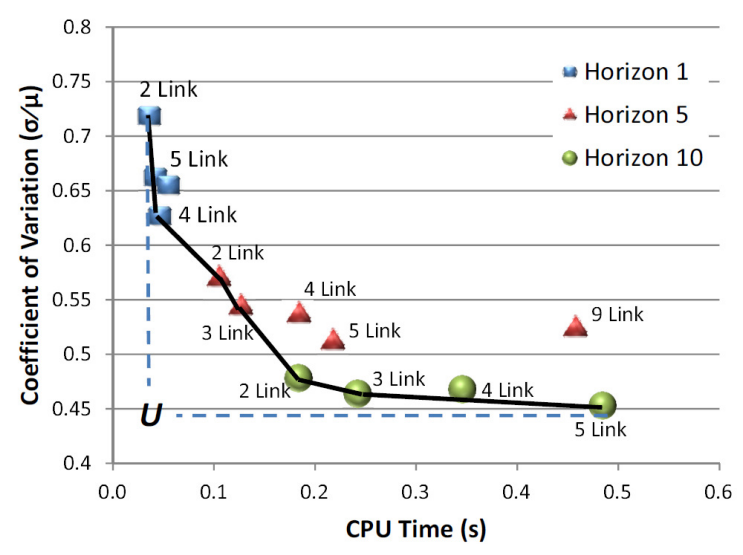

Fig. 9. Pareto plot for the CPU time and the coefficient of variation.

Based on the result obtained from the previous section, an optimal configuration of the horizon time and the number of cable links can be achieved by minimizing the CPU time, the coefficient of variation, and the number of 
failed solutions. To simplify this multi-objective problem, the number of failed solutions is neglected in the current study. Figure 9 shows a Pareto plot, in which a solid curve represents an optimum edge, i.e., a Pareto frontier, and the "U" represents a Utopia point, depicting the lowest coefficient of variation and CPU time that can be expected. In this study, an optimal configuration can be selected as a 5 -second horizon time and a 3-link cable model by equally weighting the CPU time and the coefficient of variation.

C. Further analysis of the result

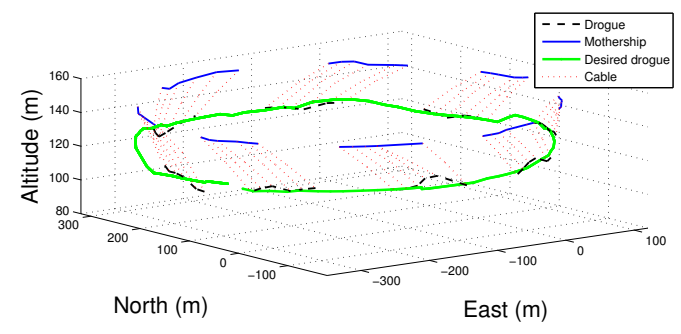

Fig. 10. Horizon segments in the recursive estimation at 10, 20, 30, 40, 50, 60, 70 and 80 seconds, respectively.

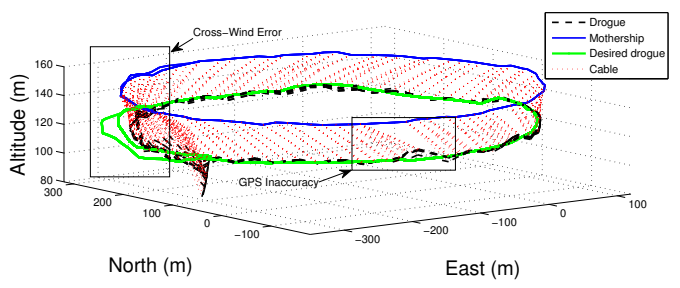

Fig. 11. Moving horizon estimation can be visualized by a successive set of time horizons where the parameters are optimized to match experimental data subject to the equations of motion.

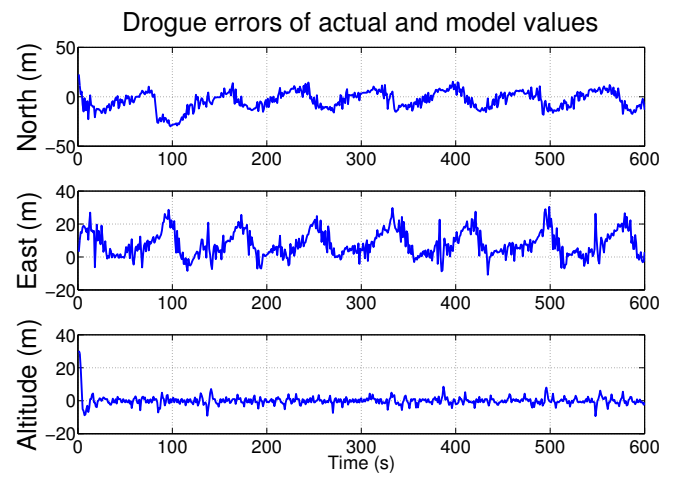

Fig. 12. Drogue errors of the measured and model values in North, East and Altitude, respectively. 
An analysis is conducted in this section to investigate the influence of the accuracy of the flight test data to the estimation result. Figure 10 shows horizon segments in the recursive estimation at 10, 20, 30, 40, 50, 60, 70 and 80 seconds, respectively. The cumulative set of 100 successive steps, each of 5 seconds, is displayed in Figure 11. Some visible discrepancy can be seen in the plots. For example, at coordinates (-150 North, -200 East) in Figure 11, the mothership appears to suddenly proceed further than the other location, but does not move for a while. This measurement inaccuracy causes the drogue to swing up from the measured flight path. To compensate for these types of measurement inaccuracies, a 2-meter deadband is given to the GPS measurement as previously described in (20). The deadband prevents state and parameter adjustments as long as the model predictions are within this region. Figure 12 shows errors of measured and model values of the drogue coordinates in the north, east and altitude directions, respectively. As shown previously in Figure 5, the horizontal wind velocity is in the north-east direction, therefore the largest error occurs when the system transitions from a cross-wind to a tail-wind. The measured values indicate that the drogue swings further out than the predicted trajectory.

\section{Estimation of the vertical wind velocity and the Young's modulus}

This section further investigates whether parameters like the Young's modulus and variables like the wind velocity in the vertical direction can be estimated by the proposed approach. Using a 3-link model and a 5-second horizon time, estimates of the Young's modulus oscillate between 200 and $400 \mathrm{MPa}$, and estimates of the vertical component of the wind velocity oscillate $\pm 3 \mathrm{~m} / \mathrm{s}$. Increasing the horizon time does not attenuate the oscillation but only increases the CPU time. The results produce less applicable solutions than those of $C_{D_{d r}}$.

To investigate the qualification of parameters that can be estimated using the proposed approach, Table II lists the sensitivity of the aerodynamic drag coefficient of the drogue, the Young's modulus of the cable, the applied cable length, and the vertical wind velocity, relative to the drogue Down coordinate. It can be seen that sensitivities of the Young's modulus and the vertical wind velocity relative to measured variables (drogue Down coordinate) of the model is much smaller than the other two parameters, so a solver has to make larger changes in the Young's modulus and vertical wind velocity to reconcile the model with the actual data.

TABLE II

SENSITIVITIES WITH $1 \%$ CHANGE IN PARAMETERS.

\begin{tabular}{|c|c|}
\hline Parameters $\backslash$ Measured variables & $p_{N}^{d}(\mathrm{~m})$ \\
\hline \hline Drag coefficient & -1.761 \\
\hline Young's modulus & -0.008 \\
\hline Applied cable length & 0.264 \\
\hline Vertical wind velocity & -0.016 \\
\hline$\left(p_{N}^{n}, p_{N}^{e}, p_{N}^{d}\right)^{T}$ is drogue 3-D coordinates in NED frame. \\
\hline
\end{tabular}



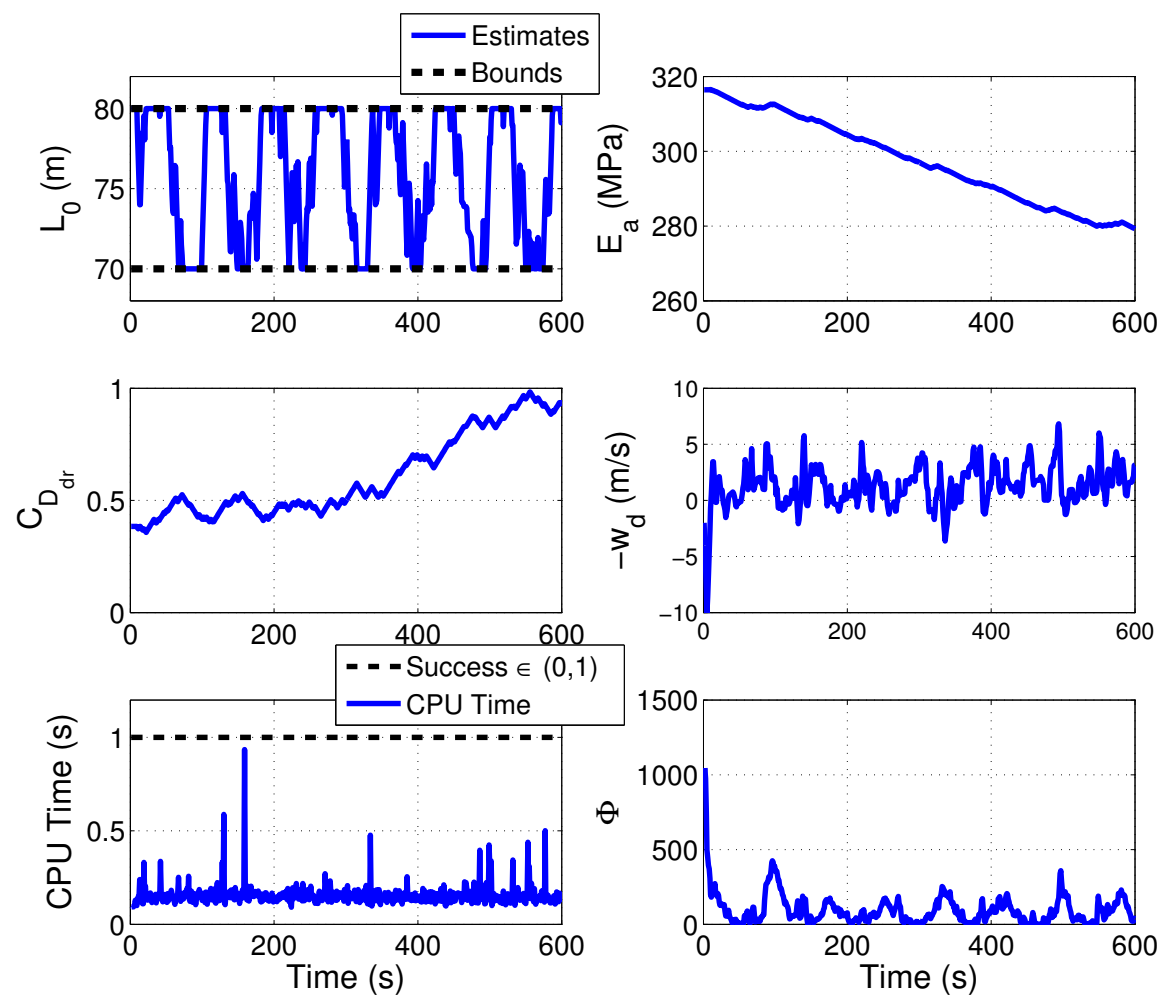

Fig. 13. Estimation results with multiple parameters.

\section{E. Multivariate parameter estimation}

Studies of the single parameter estimation in Sections IV-B, V-A, and V-D reveal that certain parameters like the applied cable length and the drag coefficient of the drogue can be estimated one at a time by matching the locations of the drogue in flight test and simulation. In this section, multiple parameters are simultaneously estimated to further investigate the applicability of the proposed approach. Based on the result in Figure 9, a 3-link cable and a 5 -second horizon time are selected in simulation.

Parameters and variables selected for estimation include the applied cable length, the Young's modulus, the drag coefficient of the drogue, and the wind velocity in the vertical direction. Based on the knowledge of the physical system, any variation after an initial adjustment in parameters, such as the Young's modulus and the drag coefficient, of which are expected to remain somewhat constant throughout the simulation, indicates that there may be either a structural error of the model or that the parameters are being adjusted for measurement inaccuracy. Other parameters such as the cable length and the wind velocity are expected to vary about a median value.

Figure 13 shows the results of parameter estimates as well as the CPU time and objective function values over a $600 \mathrm{~s}$ window. Upper and lower bounds for the cable length estimates are set to 80 and 70 meters, respectively, to prevent unrealistic values from influencing other parameter estimates. It can be seen that the applied cable length 
fluctuates with each circulation of the system whereas parameters such as the drag coefficient of the drogue and the Young's modulus are independent of angular position. Estimates of the vertical wind velocity fluctuates typically between $5 \mathrm{~m} / \mathrm{s}$ and $-2 \mathrm{~m} / \mathrm{s}$ indicating a possible slight thermal uplift. In addition, the objective function trend shows that the error is larger on the north-west side of the trajectory.

\section{CONCLUSiON}

In this paper, a real-time optimal estimation for parameters in a towed cable system is presented using moving horizon estimation (MHE). Preliminary experimental results reveal the capability of the algorithm to obtain an estimation for the applied cable length in real-time. Given the success of the preliminary flight test, a further offline study demonstrates that multiple parameters can be updated during flight with a rigorous nonlinear model. The framework is a reliable method to obtain parameter values that accurately predict the future motion of a system. Consistent parameter values with predictive certainty are important for optimal control where a trajectory is planned and optimized based on a predictive model.

A detailed offline simulation analysis used experimental data to further investigate the configuration of the optimization for the best combination of computation burden, model complexity, and frequency of failed solutions. The solutions for this multi-objective optimization problem is illustrated by a Pareto plot, which shows that given the current hardware and software platform, a 5-second time horizon and a 3-link cable model is an optimal configuration. In addition, a sensitivity analysis reveals that the objective function cannot be significantly minimized, except with large and unrealistic changes in certain parameter values. The sensitivity analysis suggests which parameters can align the model to measured values and which parameters have little influence on model predictions and should not be adjusted. The multivariate parameter estimation result presented the ability of the proposed estimation strategy to simultaneously adjust multiple parameters.

\section{ACKNOWLEDGMENT}

This research was supported by the Air Force Office of Scientific Research under STTR contract No. FA 955009-C-0102 to Procerus Technologies and Brigham Young University.

\section{REFERENCES}

[1] R. A. Skop and Y. Choo, "The configuration of a cable towed in a circular path," Journal of Aircraft, vol. 8, no. 11, pp. 856-862, November 1971.

[2] P. Trivailo, D. Sgarioto, and C. Blanksby, "Optimal control of aerial tethers for payload rendezvous," in Control Conference, 2004. 5th Asian, vol. 1, July 2004, pp. 396-404 Vol.1.

[3] P. Williams and W. Ockels, "Dynamics of towed payload system using multiple fixed-wing aircraft," AIAA Journal of Guidance, Control, and Dynamics, vol. 32, no. 6, pp. 1766-1780, November-Decemeber 2009.

[4] P. Williams, B. Lansdorp, and W. Ockels, "Optimal cross-wind towing and power generation with tethered kites," AIAA Journal of Guidance, Control, and Dynamics, vol. 31, no. 1, pp. 81-93, 2008. 
[5] C. Lambert and M. Nahon, "Stability analysis of a tethered aerostat," Journal of Aircraft, vol. 40, no. 4, pp. 705-715, 2003.

[6] J. W. Kamman and R. L. Huston, "Modeling of variable length towed and tethered cable systems," AIAA Journal of Guidance, Control, and Dynamics, vol. 22, no. 4, pp. 602-608, 1999.

[7] Y. Turkyilmaz and O. Egeland, "Active depth control of towed cables in 2D," IEEE Conference on Decision and Control, pp. 952-957, December 2001.

[8] P. Williams, “Optimal terrain-following for towed-aerial-cable sensors," Multibody Syst Dyn, vol. 16, pp. 351374, 2006.

[9] K. Ro and J. W. Kamman, "Modeling and simulation of [Hose-Paradrogue aerial refueling systems," AIAA Journal of Guidance, Control, and Dynamics, vol. 33, pp. 53-63, 2010.

[10] I. Kolodner, "Heavy rotating string - a nonlinear eigenvalue problem," Communications on Pure and Applied Mathematics, vol. 8, pp. 395-408, 1955.

[11] N. Nakagawa and A. Obata, "Longitudinal stability analysis of aerial-towed systems," Journal of Aircraft, vol. 29, no. 6, pp. 978-985, 1992.

[12] P. Williams and P. Trivailo, "Dynamics of circularly towed cable systems, part 1: Optimal configurations and their stability," AIAA Journal of Guidance, Control, and Dynamics, vol. 30, no. 3, pp. 753-765, May-June 2007.

[13] J. Genin, S. J. Citron, and R. R. Huffman, "Coupling of longitudinal and transverse motions of a flexible cable in a uniform flow field," The Journal of the Acoustical Society of America, vol. 52, no. 1B, pp. 438-440, 1972.

[14] J. E. J. Cochran, M. Innocenti, T. S. No, and A. Thukral, "Dynamics and control of maneuverable towed flight vehicles," AIAA Journal of Guidance, Control, and Dynamics, vol. 15, no. 5, pp. 1245-1252, 1992.

[15] P. Williams, P. Lapthorne, and P. Trivailo, "Circularly-towed lumped mass cable model validation from experimental data," in AIAA Modeling and Simulation Technologies Conference and Exhibit, ser. AIAA 20066817, Keystone, Colorado, August 2006.

[16] L. Sun and R. W. Beard, "Towed body altitude stabilization and states estimation in aerial recovery of micro air vehicles," in AIAA Guidance, Navigation, and Control Conference, Toronto, Ontario Canada, August 2010.

[17] J. F. Henderson, J. Potjewyd, and B. Ireland, "The dynamics of an airborne towed target system with active control," Proceedings of the Institution of Mechanical Engineers, Part G: Journal of Aerospace Engineering, vol. 213, no. 5, pp. 305-319, 1999.

[18] L. Sun, R. W. Beard, M. B. Colton, and T. W. McLain., "Dynamics and control of cable-drogue system in aerial recovery of micro air vehicles based on Gauss's principle," in American Control Conference, St. Louis, MO, USA, June 2009, pp. 4729-4734.

[19] L. Sun, R. W. Beard, and M. B. Colton, "Motion planning and control for mothership-cable-drogue systems in aerial recovery of micro air vehicles," in American Control Conference, Baltimore, MD, USA, 2010, pp. $2101-2106$.

[20] L. Sun and R. W. Beard, "Towed-body trajectory tracking in aerial recovery of micro air vehicle in the presence 
of wind," in American Control Conference, San Francisco, CA, USA, 2011, pp. 3209-3214.

[21] M. Colton, L. Sun, D. Carlson, and R. Beard, "Multi-vehicle dynamics and control for aerial recovery of micro air vehicles," Int. J. Vehicle Autonomous Systems, vol. 9, pp. 78-107, 2011.

[22] L. Sun, J. D. Hedengren, and R. W. Beard, "Optimal trajectory generation using model predictive control for aerially towed cable systems," AIAA Journal of Guidance, Control, and Dynamics, vol. 37, no. 2, pp. 525-539, 2014.

[23] J. W. Nichols, L. Sun, R. W. Beard, and T. McLain, “Aerial rendezvous of small unmanned aircraft using a passive towed cable system (accepted)," AIAA Journal of Guidance, Control, and Dynamics, 2014.

[24] F. S. Hover, "Experiments in dynamic positioning of a towed pipe," in OCEANS '93. Engineering in Harmony with Ocean, vol. 3, Victoria, BC, Canada, Oct 1993, pp. III484 - III490.

[25] J. M. Clifton, L. V. Schmidt, and T. D. Stuart, "Dynamic modeling of a trailing wire towed by an orbiting aircraft," AIAA Journal of Guidance, Control, and Dynamics, vol. 18, no. 4, pp. 875-881, 1995.

[26] J. Coomer, M. Lazarus, R. Tucker, D. Kershaw, and A. Tegman, “A non-linear eigenvalue problem associated with inextensible whirling strings," Journal of Sound and Vibration, vol. 239, pp. 969-982, 2001.

[27] S. Jones and J. Krausman, "Nonlinear dynamic simulation of a tethered aerostat," Journal of Aircraft, vol. 19, no. 8, pp. 679-686, 1982.

[28] B. Etkin, "Stability of a towed body," Journal of Aircraft, vol. 35, no. 2, pp. 197-205, 1998.

[29] J. E. Quisenberry and A. S. Arena, "Dynamic simulation of low altitude aerial tow systems," in AIAA Atmospheric Flight Mechanics Conference and Exhibit, vol. 1, Providence, Rhode Island, August 2004, pp. $243-252$.

[30] R. Murray, “Trajectory generation for a towed cable system using differential flatness," San Francisco, July 1996, pp. 395-400.

[31] P. Williams, "Periodic optimal control of a towed aerial-cable system in presence of cross-wind," in $A I A A$ Guidance, Navigation, and Control Conference, ser. AIAA 2006-6192. Keystone, Colorado: American Institute of Aeronautics and Astronautics, August 2006.

[32] E. Haseltine and J. Rawlings, "Critical evaluation of extended kalman filtering and moving-horizon estimation," Ind. Eng. Chem. Res., vol. 44, no. 8, pp. 2451-2460, 2005.

[33] J. D. Hedengren, K. V. Allsford, and J. Ramlal, "Moving horizon estimation and control for an industrial gas phase polymerization reactor," in American Control Conference, July 2007, pp. 1353-1358.

[34] J. Rawlings and D. Mayne, Model predictive control: theory and design. Madison, WI: Nob Hill Publishing, LLC, 2009.

[35] K. R. Muske and T. A. Badgwell, "Disturbance modeling for offset-free linear model predictive control," Journal of Process Control, vol. 12, pp. 617-632, 2002.

[36] G. Pannocchia and J. Rawlings, "Disturbance models for offset-free MPC control," AIChE Journal, vol. 49, no. 2, pp. 426-437, 2002.

[37] G. Pannocchia and E. Kerrigan, "Offset-free control of constrained linear discrete-time systems subject to 
persistent unmeasured disturbances," in Proceedings of the 42nd IEEE Conference on Decision and Control, Maui, Hawaii, December 2003, pp. 3911-3916.

[38] S. Samar, D. Gorinevsky, and S. Boyd, "Embedded estimation of fault parameters in an unmanned aerial vehicle," in Proceedings of the 2006 IEEE International Conference on Control Applications, 2006, pp. 32653270.

[39] A. Voelker, K. Kouramas, C. Panos, and E. Pistikopoulos, "Disturbance estimation via moving horizon estimation for in-flight model-based wind estimation," in 21st European Symposium on Computer Aided Process Engineering ESCAPE 21, 2011, pp. 577-586.

[40] F. Campillo and V. Rossi, "Convolution particle filter for parameter estimation in general state-space models," IEEE Transactions on Aerospace and Electronic Systems, vol. 45, no. 3, pp. 1063-1072, July 2009.

[41] G. Rigatos, "Nonlinear kalman filters and particle filters for integrated navigation of unmanned aerial vehicles," Robotics and Autonomous Systems, vol. 60, pp. 978-995, 2012.

[42] H. Soken and C. Hajiyev, "UKF-based reconfigurable attitude parameters estimation and magnetometer calibration," IEEE Transactions on Aerospace and Electronic Systems, vol. 48, no. 3, pp. 2614-2627, July 2012.

[43] S. Ghosh and S. Mukhopadhyay, "Tracking reentry ballistic targets using acceleration and jerk models," IEEE Transactions on Aerospace and Electronic Systems, vol. 47, no. 1, pp. 666-683, January 2011.

[44] J. D. Hedengren, “APMonitor Modeling Language," apmonitor.com, retrieved April, 2013.

[45] L. Biegler, "An overview of simultaneous strategies for dynamic optimization," Chemical Engineering and Processing, vol. 11, pp. 1043-1053, 2007.

[46] J. D. Hedengren, “Advanced Process Monitoring," in Optimization and Analytics in the Oil and Gas Industry, K. C. Furman, J.-H. Song, and A. El-Bakry, Eds. Springer International Series in Operations Research and Management Science, 2014 (accepted).

[47] A. Wächter and L. Biegler, "On the implementation of a primal-dual interior point filter line search algorithm for large-scale nonlinear programming," Mathematical Programming, vol. 106, no. 1, pp. 25-57, 2006.

[48] A. Cervantes and L. Biegler, "Large-scale DAE optimization using a simultaneous NLP formulation," AIChE Journal, vol. 44, no. 1, pp. 1038-1050, 1998. 